\title{
Foro Económico Mundial y Foro Social Mundial: El desafío de sentarnos a reinventar el mundo
}

KAREN Duke ${ }^{1}$

Departamento de Economía UCA, San Salvador

I na tarde de finales de noviembre, hace una década, finalizando mi segundo año de estudiante de economía, caminaba con mi bolsón hacia el edificio B, aula 34, me dirigía a mi clase de Sistemas Económicos Comparados. Era costumbre de mi profesor escribir en el pizarrón, con yeso blanco y amarillo, el tema que se desarrollaría durante la sesión correspondiente, tal como decía él "a manera de agenda". Ese día al entrar, el escribía "el hombre de Davos y el hombre de Porto Alegre". Con el tiempo, me di cuenta que el seguimiento de los foros, cumbres y convenciones mundiales era una de sus pasiones.

Al inigualable profesor de Sistemas Económicos Comparados, quien me impartió los primeros cursos de Introducción a la Economía I y II , que me hizo ver la economía como algo fascinante, que tenía abierta la puerta de su oficina para atender las dudas de los aprendices, que tenía tiempo de conversar de cosas académicas y de cosas cotidianas; siempre con un consejo oportuno, guiándome como alumna, instructora, y cómo segundo lector de mi tesis, quien me motivó a estudiar el mundo "abriendo la ventana de la casa para ver hacia afuera". Dedico este artículo a quien siempre estuvo escudriñando la realidad, con disciplina, empeño y mucha reflexión:

Al Padre lbisate S.J.

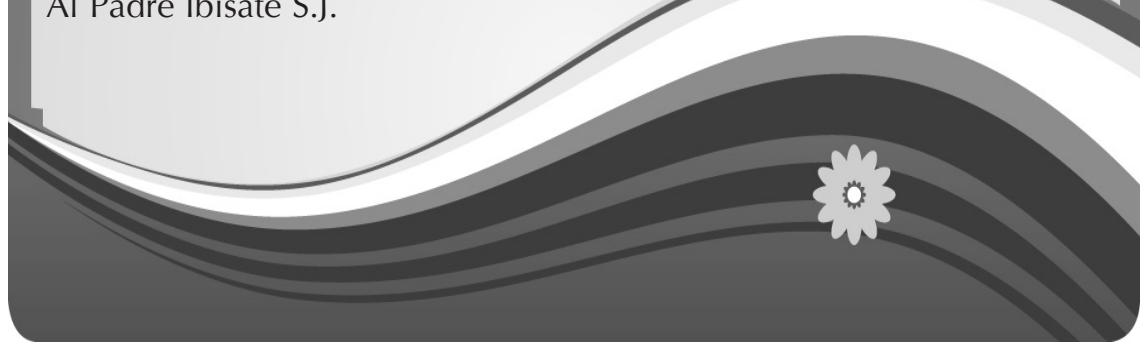


En este sentido, quiero plantear algunas ideas claves sobre la configuración del Entorno Económico Mundial desde dos perspectivas: una, desde el Foro Económico Mundial y otra, sobre el Foro So- cial Mundial; dos foros distintos y distantes, en cuanto a lugar, ubicación, cualidades y forma de ver al mundo; por lo tanto en la selección de los problemas urgentes a nivel mundial.

\section{Foro Económico Mundial (FEM)}

\section{a. Contexto}

¿Qué hacen más líderes de alrededor de cien países a más de mil metros de altitud en pleno invierno suizo?

El FEM se autodefine como una organización internacional independiente, comprometida a mejorar el estado del mundo incorporando a las empresas, académicos, líderes políticos y sociales para ir dando forma a las agendas globales, regionales e industriales. Es una fundación con sede en Ginebra conocida por su asamblea anual en Davos, Suiza, donde se busca fomentar el debate y reunirse con personajes claves a nivel internacional, político y empresarial.

El FEM fue pensado por primera vez en enero de 1971, cuando se reunieron un grupo de líderes empresariales europeos junto con el alemán Klaus M. Schwab (profesor de economía en Suiza), quien presido la reunión que tuvo lugar en Davos, y quien tuvo la visión de lo que se convertiría en el Foro Económico Mundial (en 1987), que inicio como el Foro de Gestión Europea ${ }^{2}$.
Lo que comenzó como una serie de reuniones empresariales con un enfoque de gestión, basado en el éxito empresarial (teniendo en cuenta los intereses de los accionistas, clientes, empleados, dentro del contexto de su propio entorno) ha trascendido ahora a una reunión anual en el centro vacacional suizo con el propósito de hablar y examinar cuestiones mundiales, gubernamentales y estrategia de negocios; donde asisten personalidades, empresarios y jefes de los Estados más influyentes del mundo para ponerse al día con las nuevas tendencias y planes para el futuro.

A manera de ejemplo, el Tratado de Libre Comercio de América del Norte, que vincula a Estados Unidos, Canadá y México, fue propuesto por primera vez en una reunión formal entre líderes políticos en Davos. Eventualmente el cuaderno de trabajo del foro se ha ido expandiendo hacia la economía, política y sociedad.

Asistir a esta reunión supone un honor exclusivo. Estamos hablando de una empresa tipo que tiene un volumen de negocios con más de 
cinco mil millones de dólares y que pueda pagar los altos costos de enviar ejecutivos de empresas, a dicho Foro. Entre los niveles de membrecía el nivel básico que le permite ir a Davos cuesta unos \$52,000, junto al boleto de avión valorado en unos $\$ 19,000$, haciendo un total de $\$ 71,000$ aproximadamente, solo para estar presente en las sesiones generales.

Si se desea participar en las sesiones privadas, es necesario ser parte de un "asociado industrial" que cuesta alrededor de unos $\$ 137,000$; entre otros costos como el alojamiento, comida y transporte. Así pues, a un empresa que quiera rentar cinco cuartos durante el 2011 en las afueras de Davos, le cuesta $\$ 140,000$ por la semana. En cuanto al transporte, junto con su motorista, que organiza el FEM cuesta unos $\$ 10,000$ a la semana. Entre otros costos que me parecen ofensivos mencionar. (New York Times, 2011).

¿Quiénes asisten entonces? Claramente, personalidades de prestigio del mundo de los negocios y la política. Así pues, el FEM de 2005 contó con la asistencia de jefes de corporaciones como los gigantes de computación Michael Dell y Bill Gates, John Chambers de Cisco, Nandan Nilekani de Infosys, Phillip Knight de Nike y Sir Martin Sorrell de la multinacional de la publicidad WPP. La lista de políticos incluyó al presidente de Irán, Mohammed
Jatamí; el vicepresidente de EE.UU., Dick Cheney; el Secretario General de Naciones Unidas, Kofi Annan; el presidente de Pakistán, Pervez Musharraf y el jefe de la Organización Mundial del Comercio, Supachai Panitchpakdi, entre otros.

Entre los temas centrales que se han tratado en la última década se encuentra: Internet y la ingeniería genética. ¿Cómo mantener el crecimiento y crear puentes que termine con las divisiones?: un marco de acción para el futuro global. El liderazgo en tiempo de fragilidad: una visión para un futuro común. Construyendo confianza. Asociarse para la Prosperidad y la Seguridad. Decisiones contundentes para los tiempos difíciles. Compartiendo seguridad y prosperidad. El imperativo de la creatividad; por mencionar algunos temas centrales.

En 2010, el FEM cumplió cuarenta años de trabajo, dándose cita nuevamente con más de dos mil quinientos líderes de negocios, gobierno y academia. Se reunieron para tratar el lema de Davos 2010: Mejorar el estado del mundo: repensarlo, rediseñarlo y reconstruirlo, participando en más de doscientas veinte sesiones de trabajo, tratando temas como: Reconstruyendo la economía; ¿Podrá India satisfacer las expectativas globales?; América Latina: Enfrentando el desafío democrático; De Copenhague a México: ¿qué sigue?.; El programa de género: Poniendo en práctica 
la equidad; Replanteamiento de la asistencia humanitaria: Implicaciones para Haití, entre otros.

Ya que es un acontecimiento mundial, un Foro que se compromete a sí mismo a mejorar el estado del mundo, entre los puntos centrales que resaltaron en 2010 fueron: la ofensiva de los banqueros contra la reforma del sistema financiero internacional -a propósito del plan anunciado por Barak Obama- los problemas que arrastraban a Grecia, el rol de China, los niveles de deuda de cuenta corriente y problemas de competitividad, de economías como Italia, España, Irlanda o Portugal.

El 31 de enero de 2010, Davos concluye con un consenso general sobre la fragilidad de la recuperación, pero sin un acuerdo sobre cómo estimular el crecimiento del empleo y prevenir otra turbulencia económica global. Por su parte, el Presidente de la Junta Gerencial del banco alemán Deutsche Bank, Josef Ackermann, reconoció a los actores económicos más importantes por ampliar eran el G-8 y el G-20. En esa oportunidad, el funcionario afirmó que debería haber un Grupo Empresarial de los 20 para colaborar con ellos y enfocarse en asuntos de negocios.

Un año después, a nivel mundial, nuevamente se presenta la búsqueda de dirección, cada vez más urgente, no solo por el tema de integración económica y globalización, sino también, por las nuevas configuraciones geopolíticas y económicas. De hecho, una tesis expuesta por el profesor de Economía en la Universidad de Nueva York, Ian Bremmer y Nouriel Roubini, presentaba un nuevo orden mundial que se denomina "el mundo del G-Cero", donde se planteaba que a raíz de la crisis financiera, Estados Unidos ya no podría ser el líder de la economía global, y ninguna otra nación, o grupo de naciones, tenía el peso político y económico para sustituirlo. De acuerdo a su planteamiento, Estados Unidos no poseía los recursos para continuar asegurando un panorama de estabilidad en el que prosperasen el libre mercado, el libre comercio y la movilidad del capital. Por su parte, Europa se encontraba en problemas financieros y Japón estaba enfrentando problemas nacionales complejos, políticos y económicos. China, por su parte, no parecía tener interés en aceptar las cargas que vienen con el liderazgo internacional.

Previo a la reunión de 2011, uno de los planteamientos fuertes era que la economía global se recuperaría en 2011. Pero en realidad solamente China, India y Brasil habían presentado recuperaciones veloces. Tal como lo planteaba, BBC Mundo en una nota publicada el 26 de enero: "...en Davos se esperaba un fuerte crecimiento en Estados Unidos y Alemania, pero se cree que la mayoría de las economías 
avanzadas sólo registrarán pocos progresos".

Durante Davos 2011 se presentan puntos de vista interesantes. Por cuestiones de espacio, presento sólo tres de ellas: Nicolas Sarkozy, presidente de Francia, enmarcó su visión para el G-20 señalando que "Estamos 11 años en el siglo XXI, sin embargo, siguen funcionando con las reglas del siglo XX." Hemos entrado en una época en que cada vez es más importante hablar y escucharse unos a otros, para identificar el interés colectivo común, y encontrar nuevos caminos de pensamiento que nos ayuden a construir el futuro.

Más adelante en un panel conformado por McKinsey, director de Dominic Barton y James Dimon, CEO de JPMorgan Chase, se concluyó que la economía mundial se enfrenta a muchos retos, entre ellos la carga de la deuda soberana, el crecimiento demográfico y la rápida urbanización que representan un riesgo para el desarrollo, pues agotan los recursos naturales. En Occidente, la pérdida de empleos y la deflación de los salarios amenazan la inestabilidad social al igual que el espectro cada vez mayor de desempleo de los jóvenes.

La presidenta y consejera delegada de PepsiCo, Indra Nooyi, dijo que los empresarios deben ser enviados de nuevo a la escuela para ser reeducados para pensar más allá de la obtención de beneficios. El éxito de gestión se ha definido como un modo tan estricto en el sentido de maximizar las ganancias para los accionistas, sin tener en cuenta los objetivos a largo plazo, tales como la sostenibilidad. La mentalidad empresarial aún se refiere a la adopción de la sostenibilidad como una cuestión de resolver los conflictos o compensaciones. Resalto una frase textual de Nooyi: "Creemos que los beneficios a corto plazo y la sostenibilidad a largo plazo no son mutuamente excluyentes".

Una particularidad interesante, en Davos 2011, fue que por primera vez en la historia introdujo una cuota de género, exigiendo que al menos uno de cada cinco delegados fuese una mujer, medida aplicada a la participación de empresas consideradas como "socios estratégicos". La economista Saadia Zahidi ha sido una de las impulsoras de la introducción de cuotas para mujeres dentro del FEM. Sin embargo, las mujeres que asistentes a este FEM se encuentran en un lugar peculiar: son miembros de dicha élite, pero miembros relativamente recientes, con un estatus de minoría.

Según una nota publicada por la BBC Mundo, el 13 de enero de 2011; entre 2001 y 2005, el porcentaje de participación femenina en la FEM fue entre 9 y $15 \%$ del total. Mientras que para 2011, la intervención de las mujeres cerró con un $16 \%$ solamente. 
Entre la organización, el equipo de liderazgo del FEM se encuentra escalonado por cuatro niveles de importancia: un presidente fundador, un primer equipo de directores superiores compuesto por siete hombres, un segundo equipo de directores principales compuesto por 17 personas (35\% son mujeres) y un tercer grupo de directores compuesto por 25 personas $(60 \%$ son mujeres). Parece existir una relación inversa entre rangos superiores $y$ presencia de mujeres o bien una relación directa entre grados inferiores y presencia de mujeres.

¿Cómo cierra Davos 2011? Con preocupaciones reflejadas en un comunicado publicado en su sitio: "El tema de este año fue de normas compartidas para la nueva realidad, lo que refleja el hecho de que vivimos en un mundo que es cada vez más complejo e interconectado, pero también está experimentando una erosión de los valores y principios comunes". ¿Qué valores y qué principios se entienden como comunes? ¿En el mundo? ¿En la sociedad? ¿Entre las empresas?

Entre las evidencias finales se presentan a China e India como economías claramente emergentes y con fuerza económica y política, así como la preocupación por los recursos y la salud a nivel global.

El FEM finaliza centrado en las distintas amenazas a nivel global, por una parte el tema político, con el ojo en Túnez y Egipto; y por otra en el tema de los recursos y la escasez de los mismos. Así también, el problema del Euro y erradicación de enfermedades. Mientras que una década atrás el tema central fue la "reconstrucción de la confianza", parecía que diez años después otro tipo de confianza se estaba perdiendo...

\section{b. Davos 2012. ¿Qué nos deja?}

Este año el FEM, como es costumbre, es "inaugurado" por manifestantes que acampan en las calles Davos protestando en contra del FEM, seriamente preocupados la desigualdad de ingresos y el sistema. En esta oportunidad con tiendas, marchas sin autorización (como parte de los beneficios del clima, con campamentos en forma de iglúes para pedir más ayuda para los más pobres), con peticiones anticapitalistas, para ser expuesta a la "gente importante" de los negocios y de la política mundial cuando se dieran presencia en dicha reunión anual.

Davos, que convoca a políticos, presidentes de bancos centrales y líderes empresarios, se ha convertido en un sinónimo de la globalización, en tanto a la dinámica y la forma de hacer las cosas en el mundo, que ha generado la urgente la necesidad de examinar de cerca el mundo empresarial, pero también a los gobiernos. A medida que el mundo experimenta cambios trascendenta- 
les, es necesario para las empresas y gobiernos repensar su papel y sus responsabilidades hacia la sociedad, la posición de sí mismas como un líder activas en el entorno económico actual.

Estos manifestantes, presentes año con año, pese al frío y las nevadas, buscan mandar mensajes a los organizadores y participantes de este foro, un poco de acercamiento a las deficiencias en el mundo actual. El profesor Schwab parece haber recibido un poco de estos mensajes, de acuerdo a Tim Weber en BBC, el futuro de las élites políticas y económicas "están en peligro de perder por completo la confianza de las generaciones futuras".

A propósito de esta reflexión, quisiera recordar la existencia de un estudio mundial publicado pocos días antes del comienzo de la edición 2011 de Davos: El Barómetro de Confianza que Edelman realiza cada año, en 23 países del mundo y que mide los índices de confianza que la población tiene principalmente en el tejido empresarial. El indicador sugiere una atención importante hacia la confianza del público, no sólo en los negocios, sino también en los gobiernos.

Se presenta también la transformación de la confianza, de una estructura cuadrangular, que tiene que ver con: 1) Control de la información, 2) Independencia 3) Centrarse exclusivamente en el beneficio 4)
Proteger la marca. Relacionado hacia una estructura triangular en donde se centra en 1) Transparencia (cómo) 2) Compromiso (dónde) y 3) Beneficios con propósito (qué).

Dicho estudio, concluye en cuatro elementos para reflexionar: 1) Las empresas deben alinear los beneficios y el propósito para el beneficio social, 2) Actual panorama de los medios, incrementa el escepticismo y se requiere de múltiples voces y canales, 3) Existe una demanda de autoridad y la rendición de cuentas, establecer nuevas expectativas para el liderazgo empresarial y 4) La confianza es un agente protector y conduce a beneficios tangibles, la falta de confianza es obstáculo para el cambio.

En los preparativos realizados para Davos 2012 el tema de la confianza o desconfianza reaparecía en distintas reflexiones, una y otra vez: ¿El capitalismo está funcionando? ¿Vamos a crecer de nuevo? ¿Tiene futuro el capitalismo? ¿Es apto para el siglo XXI? ¿Habrá algo que cambiar? ¿Se tratará de dar respuestas absolutas y estandarizadas para todos?

Habrá que preguntarse si las respuestas que se dieron son las que realmente debieron y deben guiar el rumbo del mundo. Realizar una evaluación sobre ¿cómo se ha hecho hasta hoy? ¿Quiénes han sido los beneficiados? $Y$ analizar se trata de las alternativas más acertadas 
para el bien de la sociedad mundial. Estas reflexiones, presentes tanto en las sesiones organizadas por el Foro, como en muchos eventos organizados por bancos, grupos industriales y gigantes corporativos; aparecen siempre, de una manera u otra, preguntas incómodas, incluso cuando son pensadas en contextos, coyunturas y estructuras específicas como la euro zona, el sector financiero, la pobreza, la desigualdad, la responsabilidad corporativa y el surgimiento de China.

En 2012, el Foro se realizó entre el 25 y el 29 de enero, con la intención de buscar nuevas medidas para afrontar la crisis económica mundial. El tema central "la gran transformación: dando forma a nuevos modelos." Con alrededor de 2600 líderes, de cien países, en más de 260 sesiones se abordaron los considerados desafíos más grandes del mundo, un mundo agitado y presionado para dar soluciones a los problemas que aquejan a la humanidad.

Entre las personalidades se encontraban Aung San Suu Kyi, (Liga Nacional para la Democracia, Birmania), Shara Burrow (Confederación Sindical Internacional) David Cameron (Primer Ministro del Reino Unido), Cristina Lagarde (Fondo Monetario Internacional), Ban Kimoon (Naciones Unidas), Thomas Friedman (New York Times), Stephen Rocha (Universidad de Yale), Mario Draghi ( Banco Central Eu- ropeo) Gordon Brown (Primer Ministro del Reino Unido 2007-2010) Yasuchika Hasegawa (Farmacéutica Takeda), Angela Merkel (Canciller alemana), Bill Gates (Fundación Bill y Melinda Gates) Sheryl Sandberg (Facebook), Christine Lagarde, (Ministra de Economía Finanzas e industria en Francia, y Miembra de la junta del Foro Económico Mundial), Joseph E. Stiglitz (Nobel de Economía y Profesor de Columbia University, USA) entre otros.

Arranca la $42^{\mathrm{a}}$ edición del FEM con un debate sobre el capitalismo, en el cual se analizó la situación del euro y la crisis económica global. Los organizadores programaron debates sobre el futuro del sistema capitalista, en lo que se reflexionó alrededor de la siguiente interrogante: ¿Está el capitalismo del siglo XX fallando a la sociedad del siglo XXI?

Así también, se trataron las preocupaciones sobre disparidades del ingreso, sesiones interactivas sobre los recursos hídricos y seguridad alimentaria, buscando analizar nuevos modelos económicos. Sin duda alguna estos elementos marcaron los debates en Davos, llegando a una reflexión interesante comentada por el profesor alemán de Economía Klaus Schwab. "No se pueden resolver los problemas con modelos superados".

Una cuestión a la que se le dio relevancia fue la crisis económica e institucional de la Unión Europea 
(UE) que pone en riesgo a los países miembros de la zona Euro que comparten al Euro como moneda común. En el discurso de apertura, Angela Merkel motivó a Europa a seguir adelante con reformas políticas estructurales si realmente se busca resolver el problema de su deuda soberana, cuyas soluciones están encaminadas a una auténtica movilidad laboral en Europa, derechos de pensión y seguro médico. Asimismo, señaló que era necesario, en el momento oportuno, combatir los puntos débiles que arrastra la crisis económica mundial. Hubo un llamado a los países del G20, para trabajar en la regulación de los mercados financieros y la importancia de asegurar el libre comercio.

Por otra parte, Klaus Schwab, en su discurso de apertura mostró tres perspectivas deseadas: la búsqueda de soluciones constructivas y nuevos modelos; la vinculación de sociedad para alcanzar una visión constructora en un largo plazo; y la unión conjunta necesaria para conseguir un apoyo sobre los valores compartidos.

Políticos, empresarios y académicos, opinaron sobre América Latina, y aun cuando hubieron elogios a la estabilidad macroeconómica en países de la región, también se advirtió claramente que aún no se han tendido las bases para un crecimiento sostenible en el futuro. Es posible que sea de las pocas ocasiones en que la situación de la región es analizada de forma positiva, frente al cúmulo de problemas por resolver, concentrados en las economías de la Unión Europea, atrapadas en una crisis de deuda soberana.

En este sentido, se trajeron a la mesa los problemas estructurales, sobre la baja productividad en América Latina y las vinculaciones con el problema de la informalidad, presente en distintas partes del mundo; los altos precios de las materias primas en los mercados internacionales y cómo esto es un termómetro fundamental del crecimiento; el reforzamiento de los sistemas financieros nacionales que permitan soportar la crisis europea; las inversiones en la extracción de recursos naturales, que es una cuestión que ha originado protestas y levantamientos populares en la historia reciente de varios países latinoamericanos.

¿Qué nos deja Davos 2012? Temor en los medios financieros y políticos a que la crisis de la deuda en la zona euro, considerada como "el mayor problema económico y el mayor riesgo a nivel mundial" y que el resto del mundo se contagie. Entendiendo que el problema tarde o temprano afectará a la comunidad mundial. Precisamente en este sentido, Christine Lagarde, advirtió "No es sólo una crisis de la Eurozona, es una crisis que podría tener efectos colaterales, efectos secundarios, en todo el mundo" "Lo que he visto y 
lo que el FMI ha visto en los números y las previsiones es que ningún país es inmune y todo el mundo tiene interés en asegurarse de que esta crisis se resuelva adecuadamente" ${ }^{\prime \prime}$.

La última encuesta realizada por la firma de relaciones públicas Edelman, cuyo presidente, Richard Edelman, habló en la sesión de liderazgo empresarial, reveló que la confianza en el negocio se había caído de $56 \%$ a $53 \%$ de los encuestados ¿Por qué la disminución de la confianza? Existe una percepción creciente de que el negocio va mal y que se explota más de lo que contribuye al desarrollo de la sociedad.

Esta edición nos recuerda análisis de problemas recurrentes, con reflexiones ya hechas en el pasado por algunos personajes claves en la discusión, como de Joseph E. Stiglitz (Nobel de Economía y Profesor de la universidad de Columbia) y el tema de la globalización: ¿La globalización ha llegado a su límite? ¿A dónde vamos?, fueron algunas de las reflexiones alrededor del debate de la globalización y ante tales preguntas el debate no se generaron respuestas tan claras. Sin embargo no hay duda que una forma más justa de la globalización debe adoptar el comercio justo, igualdad de género y un mejor acceso a lo que la sociedad puede ofrecer.

Claramente, como se nos presenta el mundo actual, el capitalismo global no está trabajando en los intereses de la mayoría de la población mundial. Habrá que analizar si en algún momento lo ha hecho. Lo cierto es que en 1997 se habló de la necesidad de comprometerse con una nueva arquitectura global. Pero no sucedió. El nivel de frustración y desesperación, incluso en países ricos como los Estados Unidos, no debe ser subestimado. Aquellos en el poder que ignoran estas realidades lo hacen bajo su propio riesgo.

Davos 2012 nos deja con reflexiones pendientes, haré mención de algunas, que a juicio personal considero importantes para darles pensamiento: En primer lugar Nouriel Roubini, (profesor de Economía en la Universidad de Nueva York, EE.UU) "Distopia -lo contrario de la utopía- es un término bastante apropiado para representar a nuestros tiempos en los que tantas utopías han desaparecido y nos enfrentamos a tantas incertidumbres y riesgos." ${ }^{4}$ Destaca el enorme aumento de la desigualdad en las economías avanzadas, en los mercados emergentes y en los países; los desafíos que vienen de la pobreza, el subempleo y el desempleo. Toda una atmósfera de inseguridad importante, que viene de los efectos de la crisis financiera implicando una reacción en cadena.

Sin duda la inestabilidad económica que conduce a su vez a la inestabilidad política y social, lleva hacia la incertidumbre, la falta de confianza que se traduce en de- 
cisiones económicas, que genera una nueva ronda de debilidad en el sistema. Es necesario tratarlo en el corto plazo y cómo dijo Roubini "A menos que se frente a la serie completa de los riesgos globales... vivimos en un mundo en el que la inter-reacción entre los riesgos sociales y políticos y los económicos y financieros producirá un aumento de la distopía" ${ }^{5}$

La segunda reflexión viene por parte de Sharan Burrow (Secretaria General de la Confederación Sindical Internacional en Bruselas), quien en una reunión sobre el futuro del capitalismo, que fue organizada por la revista Time, argumentó que la creciente desigualdad de ingresos y el alto desempleo, especialmente entre los jóvenes, son un indicador de que el sistema capitalista ha fracasado a la sociedad. La comunidad empresarial "ha perdido su brújula moral." 6 "Debemos rediseñar el modelo. Tenemos que restablecer. Detener la codicia. A menos que los empleadores y los trabajadores se sienten con los gobiernos, el sistema continuará fracasando." 7

Una tercera reflexión fue expresada por Polman (Director Ejecutivo y Delegado de la firma Unilever Reino Unido) quien estableció que "el momento de la verdad"8 ha llegado a los países que han estado gastando más allá de sus medios, se ven obligados a medidas de austeridad que amenazan con empujar su economía en una espiral deflaciona- ria y como parte fundamental de las consecuencias el desempleo juvenil se está disparando.

La cuarta reflexión proviene de Josette Sheeran, Directora ejecutiva del Programa de Alimentos Mundial, quien nos recuerda que "el $40 \%$ de los alimentos que se producen en el mundo se pierde o se desperdicia. Para alimentar a la creciente población mundial -7,000 millones actualmente-, se necesita aumentar la producción de alimentos en un 70\% hacia 2050" De tal forma que, aumentar la producción de alimentos, mejorar su distribución y reducir los precios, al tiempo que se respeta el medio ambiente, son parte de los grandes desafíos para erradicar el hambre y alimentar a una población, que cómo bien nos ilustró Thomas Malthus, necesidades que crece exponencialmente.

Aun cuando el FEM se concentró en la crisis de la Euro zona, también lo hizo en la crisis del capitalismo, pero al igual que muchos otros temas, no se encontraron nuevos caminos. Incluso la crisis que inaugura el tercer milenio no tiene un documento estructurado de referencia, como lo fue el Consenso de Washington para la década de los 90. Se busca un capitalismo para servir mejor a la sociedad, los negocios, la banca, los líderes sindicales y académicos, que resuelva los problemas de desempleo, desorden climático, desigualdad e 


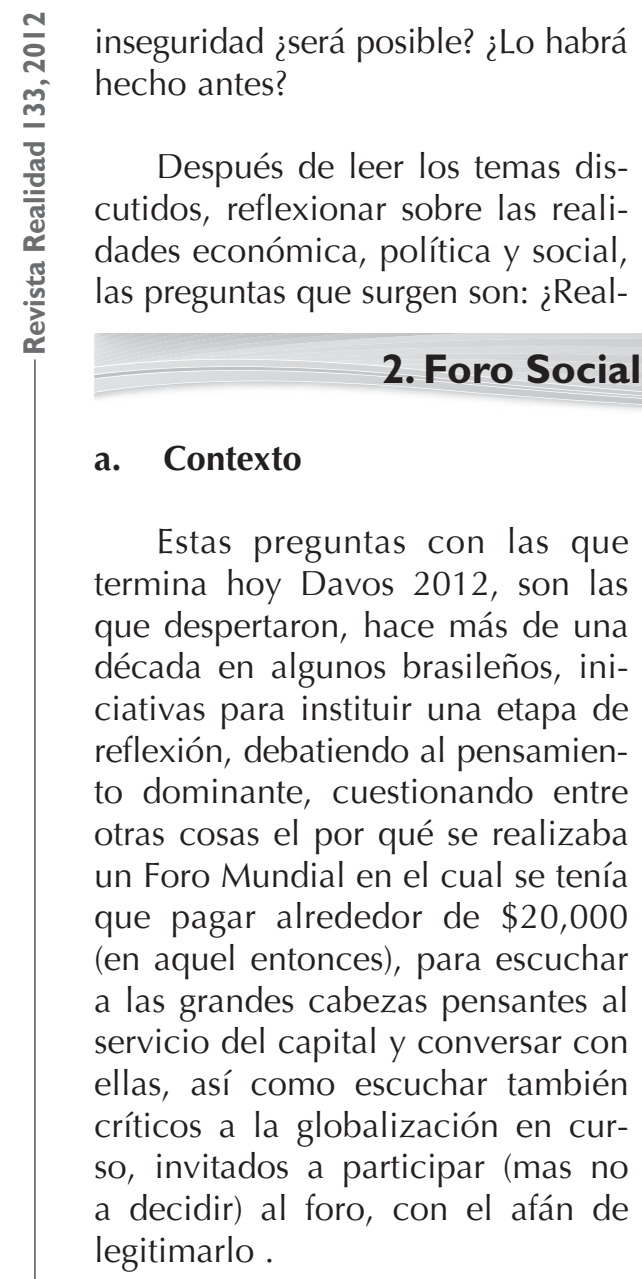

Hasta ese momento, se habían dedicado a realizar protestas y manifestaciones, pero consideraron que era momento de iniciar una nueva etapa de resistencia al pensamiento capitalista, así que además de las manifestaciones en masa y protestas, parecería posible pasar a una etapa propositiva, de búsqueda concreta de respuestas, al desafío de construir "otro mundo", en el mente este Foro trata los problemas con mayor envergadura para el mundo? ¿Quiénes deciden qué es lo importante para el mundo? ¿Se han planteado soluciones reales? ¿Se trasciende de la discusión a la realidad?

que la economía estuviese al servicio del ser humano y no al revés.

En tal sentido, se propusieron realizar un encuentro, de dimensión mundial y con la participación de todas las organizaciones que se venían articulando en las protestas masivas, orientado hacia lo social: El Foro Social Mundial un encuentro que buscó darle una dimensión simbólica al inicio de esta nueva etapa, durante los mismos días en que los grandes del mundo se encontraban en Davos de 2001.

Entre los nombres que aparecen, junto a muchos otros, Ilevando a cabo esta idea fueron Oded Grajew, Francisco Whitaker, Olivio

Dutra, Raúl Pont y Bernard Cassen ${ }^{10}$ quien propuso que el Foro se realizara en un país del considerado "tercer mundo", por su simbolismo. Fue así como Brasil estaba entre los países con mejores condiciones de acoger un Foro de este tipo, y qué mejor lugar que Porto Alegre, donde se estaban presentando una serie de experiencias democráticas y de lucha contra el neoliberalismo. 
Este Foro Social Mundial, buscaba desde aquel momento asegurar la presencia de participantes de todo el mundo, ser el inicio de un proceso de reflexión conjunta, abriendo un espacio para la búsqueda de alternativas al pensamiento único. Pero también ser el primer paso a una nueva etapa de lucha contra la sumisión del ser humano a los intereses del capital.

El Primer Foro Social Mundial, un espacio de debate democrático de ideas, reflexión profunda, formulación de propuestas, cambio de experiencias y articulación de movimientos sociales, redes, ONG y otras organizaciones de la sociedad civil que se oponen al neoliberalismo y al dominio del mundo por el capital, por cualquier forma de imperialismo. Es así que se llevó a cabo por primera vez en Porto Alegre, el FSM entre el 25 y 30 de enero (donde se esperaban 2,500 participantes) acudieron más de 4,700 delegados de 117 países.

Una asistencia total de unos 20 mil participantes, con unas 400 actividades auto gestionadas para tratar cuatro ejes fundamentales: 1) La producción de la riqueza y la reproducción social, 2) El acceso a las riquezas y a la sustentabilidad, 3) La afirmación de la sociedad civil y de los espacios públicos y 4) Poder político y ética en la nueva sociedad.
Entre las preguntas generadoras de reflexión, y que también las reflexiona ahora Davos una década después, pero sin duda desde otra perspectiva son: ¿Cómo construir un sistema de producción de bienes y servicios para todos? ¿Qué comercio internacional queremos? ¿Qué sistema financiero es necesario para asegurar la igualdad y el desarrollo? ¿Cómo garantizar las múltiples funciones de la tierra? ¿Cómo traducir el desarrollo científico en desarrollo humano? Finalizando con una proclamación que surgió en Porto Alegre, "otro mundo es posible".

Como resultado del mismo, no se presenta un documento final, pero sí hay proposiciones presentadas en la ceremonia de clausura: 1) Realizar Foros Sociales Mundiales todos los años, 2) Celebrar estos foros en la misma fecha a la del Foro Económico de Davos, 3) Constituir un Consejo Internacional del Foro Social Mundial, 4) Llevar a cabo en 2003 un Foro Social Mundial en otro país en donde hayan sido logradas las mejores condiciones de realización de los Foros en 2002, 4) Convocar en 2004 una nueva ronda de Foros en muchos países, entre otras. Finalmente, un Ilamado a la movilización, hecho por 188 organizaciones de todo el mundo, del cual cito:

"Fuerzas sociales procedentes de todo el mundo, nos hemos reunido aquí en el Foro Social Mundial de Porto 
Alegre. Sindicatos y ONG, movimientos y organizaciones, intelectuales y artistas, construimos juntos una gran alianza para crear una nueva sociedad, distinta a la lógica actual que coloca al mercado y al dinero como la única medida de valor. Davos representa la concentración de la riqueza, la globalización de la pobreza y la destrucción de nuestro planeta. Porto Alegre representa la lucha y la esperanza de un nuevo mundo posible, donde el ser humano y la naturaleza son el centro de nuestras preocupaciones."

El Ilamado termina en estos términos:

"Nuestra participación en el Foro Social Mundial ha enriquecido la comprensión de cada una de nuestras luchas y hemos salido fortalecidos. Llamamos a todos los pueblos del mundo a unirse a esta lucha por construir un futuro mejor. El Foro Social Mundial de Porto Alegre es un camino hacia la soberanía de los pueblos y un mundo justo."11

Después del primero encuentro mundial, realizado en 2001, se configuró como un proceso mundial permanente de búsqueda y construcción de alternativas a las políticas neoliberales; las cuales tendrían una dimensión internacional. En un contexto plural y diversificado, no confesional, no gubernamental y no partidario, que articulara de manera descentralizada y en red a entidades y movimientos que estarían involucrados en acciones concretas para la construcción de un mundo diferente, local o internacional; oponiéndose a toda visión totalitaria y reduccionista de la economía, del desarrollo y de la historia, así como al uso de violencia como medio de control social por parte del Estado.

En enero 2002, en Porto Alegre, en la segunda edición se presentan
50 mil participantes, entre ellos 12,274 delegados de 123 países. Se hizo un Ilamado a ampliar el FSM, con procesos y eventos nacionales, continentales, temáticos en otros. Un año más tarde, Porto Alegre, aglutina 100 mil participantes con cerca de 20.000 delegados y 1,300 actividades autogestionadas. La cuarta edición, enero 2004. Mumbai, India, con 74,126 participantes de 1,653 organizaciones de 117 países caracterizada por una fuerte presencia de movimientos "dalit" (casta de los intocables) y de representantes de organizaciones del país entero.

Primer quinquenio, enero 2005, Porto Alegre. Se presenta una marcha como apertura con 200 mil personas, 155 mil participantes registrados, 35 mil de ellos integraban 
el Campamento de la Juventud. Se implementa una nueva metodología de consulta temática, formulario por Internet que permitía identificar luchas, actividades, problemas y propuestas. Sexta edición 2006, FSM "policéntrico", descentralizado en tres ciudades de igual número de continentes: Bamako (Mali), del 19 al 23 de enero; Caracas (Venezuela), del 24 al 29 de enero, y Karachi (Pakistán), a fines de marzo.

Nairobi, Kenia, enero 2007. Una participación de 50 mil personas. Tal vez la edición más problemática y con mayores contradicciones internas. Mientras que en 2008 emana la movilización y acción global en centenares de ciudades del mundo entero. Novena edición del FSM, enero 2009, Belém do Pará, Brasil. 133 mil participantes, de 142 países. En torno a 2.300 actividades, promovidas por 5,808 entidades inscritas. Entre ellas casi 4,200 de América Latina, un poco menos de 500 de Europa y prácticamente un número similar de África fue una edición con fuerte presencia de los pueblos autóctonosoriginarios $^{12}$.

“Diez años después: retos y propuestas para otro mundo posible", fue el tema del seminario que abre las actividades del Foro Social Mundial, una década después, en Porto Alegre, Río Grande do Sul (Brasil) entre el 25 y 29 de enero. Definitivamente, el mundo había cambiado desde el $1^{\circ}$ Foro Social
Mundial celebrado en enero de 2001: guerras, crisis, nuevos movimientos de liberación, el equilibrio de poder entre las potencias hegemónicas y la geopolítica mundial.

No obstante, la identidad original del FSM permanecía como un espacio de auto-organización de las entidades y movimientos de la sociedad civil que surgen con el fin de luchar por otro mundo. Reconociendo su entorno y buscando establecer nuevos retos ante el mundo cambiante.

Inicialmente, se reflexionó sobre la evaluación de sus logros y límites, luego la dinámica de reflexión se centro en hacer una especie de diagnóstico en su más amplio sentido, evaluando de lo que ocurría en la coyuntura el planeta y la humanidad, así como los grandes retos de la civilización (tanto en el sentido de las crisis como el de las oportunidades), en la mesa puestos para todos nosotros en ese preciso momento histórico. En este sentido, se generaron cuatro mesas paralelas para profundizar sobre la situación ambiental, económica, política y social. Elementos que sentaron las bases para la nueva agenda política del Foro.

El FSM 10 años, fue un evento descentralizado, que se realizó durante todo el año en diversas ciudades del mundo. En total, más de treinta foros fueron programados, con la idea de que todos los even- 
tos estuviesen interrelacionados y contribuyeran a la construcción del próximo Foro Social Mundial, nuevamen centralizado, que tendría lugar en 2011, en Dakar, Senegal.

No obstan en el seminario de los diez años se reunieron 35.000 personas, donde se dieron encuentro representantes de 39 países, y de estos, aproximadamente el $60 \%$ eran mujeres y el $27 \%$ eran jóvenes. En coherencia con sus propuestas de origen, el FSM busca nuevamente la existencia de construcciones colectivas de todas las órdenes con el fin de buscar un mundo mejor.

Algunos de los asistentes: Christophe Aguiton (sindicalista francés, y miembro de la dirección internacional de la ONG ATTAC), Corinne Kumar (socióloga, escritora y poeta), David Harvey (geógrafo marxista británico, formado en Universidad de Cambridge), Edgardo Lander (sociólogo venezolano y docente-investigador del Departamento de Estudios Latinoamericanos de la Escuela de Sociología), Eduardo Galeano (escritor uruguayo), Gustave Massiah (economista, urbanista e analista político francés, profesora de urbanismo en París), Jamal Juma (activista palestino), Paulo Artaxo (profesor titular del Instituto de Física de la Universidad de São Paulo), Samir Amin (intelectual e economista egípcio, director del Foro del Tercer Mundo en Dakar, Senegal), Zraih Ab der Kadel (profesor de filosofía, dirigente del Foro de las Alternativas, en Marruecos), entre otros.

Su evaluación, ha sido positiva, por su impacto a nivel mundial en distintos países, por los diversos temas, relacionados con la educación, el medio ambiente, la cultura, la economía solidaria, la democracia y los derechos humanos. En definitiva, se fortaleció la participación de los jóvenes que están involucrados en las transformaciones sociales y económicas del mundo, pero también, porque las crisis económicas mundiales de los últimos años mostraron que el FSM estaba tras reflexiones bastante acertadas en tanto al agotamiento del capitalismo neoliberal.

Dakar (Senegal) y Nairobi (Kenya), fueron las ciudades africanas que recibieron el FSM 2011, una edición centralizada entre el 6 y el 11 de febrero. El retorno al continente expresó la solidaridad activa del movimiento social internacional. Inició con 10,000 asistentes, que más tarde se transformarían en 60,000 personas de distintos países del mundo.

El evento inició con una marcha que estuvo encabezada por personalidades de diversos países asistentes, entre ellas los presidentes de Bolivia y Guinea, Evo Morales y Alpha Condé, respectivamente; el ex mandatario brasileño, Luiz Inacio Lula Da Silva y Martine Aubry, líder del Partido Socialista Francés. 
En la edición 2011, se continua reflexionado como las ediciones anteriores sobre economía solidaria, comercio justo, deuda externa, reforma del sistema financiero, soberanía alimentaria, justicia climática, Derechos Humanos, explotación a niños y mujeres, militarización de los conflictos sociales, migraciones, descolonización, acceso al agua, diversidad sexual, comunicación popular, etc.

Entre los elementos interesantes propios de la edición: las rebeliones populares de Túnez y Egipto acaparaban las conversaciones de los participantes en la marcha inaugural. Más tarde, una carta mundial de migraciones y una carta de solidaridad con la lucha de las mujeres del mundo con la participación de 18 organizaciones de mujeres conscientes de que la unión de nuestras fuerzas permitirá con el tiempo provocar un cambio, reafirmaron su solidaridad y admiración por las luchas Ilevadas a cabo por las mujeres de Senegal, de África y del mundo entero.

Se reafirman la lucha en el afán de respetar y defender los principios de justicia, paz y solidaridad, y el avance en la construcción de alternativas para hacer frente a las crisis, pero en propuestas que no sean paliativas, basadas en la lógica del mercado. En este sentido, se muestran intolerantes a la persecución de la diversidad sexual, así como a las prácticas culturales que atentan contra la salud, el cuerpo y el alma de la mujer.

Durante los siguientes días se presentaron centenares de actividades auto-gestionadas en forma de talleres, conferencias y debates, que permitieron a los diversos actores de la sociedad civil mundial intercambiar ideas, reflexiones y experiencias. Por un lado hacia la lucha contra la pobreza, la miseria, las desigualdades, la precariedad, los ataques a la libertad en el mundo, para generar mejoras de las condiciones de vida y de expresión de los sectores populares directamente afectados por las políticas económicas y sociales imperantes.

Pero también, hacia el desafío de demostrar que otro mundo es posible y necesario; un mundo que actualmente pasa por la ruptura con los modos de producción, de consumo y de redistribución económica, social, ecológica, con las relaciones geopolíticas impuestas en las últimas décadas y los modelos democráticos planteados por occidente.

Esto a través de 12 ejes específicos: 1) Dignidad y diversidad; 2) Justicia ambiental; 3) Todos los derechos humanos; 4) Migraciones y el establecimiento; 5) Derecho a los saberes y medios; 6) Mundo libre; 7) Justicia económica; 8) Democracia y participación; 9) Paz y autodeterminación; 10) África y diáspora; 11) Pensando el FSM y 12) Mundo compartido. 
El FSM 2011 termina con la Declaración de la Asamblea de los Movimientos Sociales, donde afirman el apoyo y solidaridad activa a los pueblos de Túnez y Egipto; así como al mundo árabe; que busca reivindicar una real democracia y construir poder popular. Con sus luchas, muestran el camino a otro mundo, libre de la opresión y de la explotación.

Así también se presenta la solidaridad hacia a los pueblos de Costa de Marfil, de África y de todo el mundo en su lucha por una democracia soberana y participativa. Defender el derecho a la autodeterminación y el derecho colectivo de todos los pueblos del mundo. A las denuncias al rol de los agentes del sistema (bancos, transnacionales, conglomerados mediáticos, instituciones internacionales etc.), que, en búsqueda del máximo lucro al sistema capitalista, destruye la vida cotidiana de la gente, el medio ambiente y la soberanía alimentaria, incluso con métodos despreciables como la violencia sexista, la guerra, el colonialismo, las ocupaciones y la militarización de territorios.

\section{b. Porto Alegre 2012. ¿Qué nos deja?}

A manera de reflexión preparatoria del último FSM, quisiera retomar las palabras de Frei Betto:

"Como resultado de la incompetencia de un sistema que prioriza la acumulación privada de la riqueza en detrimento de los derechos humanos, sociales y ambientales, el capitalismo conoce ahora una nueva crisis. Ante ella, la reacción de los dueños del poder es la misma música de una sola nota: austeridad, recortes, aumento de impuestos y desempleo, flexibilización de las leyes laborales, congelamiento de salarios. Se salvan los bancos y perece la población. Más miseria a la vista; jóvenes sin perspectiva de futuro... flujos migratorios desordenados. Por el lado de la esperanza, y después de tres décadas de globocolonización neoliberal, las manifestaciones señalan valores positivos como la empatía ante el sufrimiento ajeno, la solidaridad, la defensa de la igualdad, la búsqueda de justicia, el reconocimiento de la diversidad y la preservación ambiental. Sin ese universo ético no hay esperanza de que se construya otro mundo posible". ${ }^{13}$

"Crisis capitalista. Justicia social y ambiental", fue el tema central abordado en Porto Alegre del 24 al 29 de enero. Propósito fundamental: la discusión de la actual crisis y su relación con la crisis ambiental y el reiterado fracaso de las cumbres que han buscado renovar los acuer- 
dos del tratado de Kyoto, la última de las cuales se realizó en diciembre pasado en la ciudad sudafricana de Durban y que termino en fracaso al no contemplar acuerdos vinculantes con relación a temas como el calentamiento global y en general con los temas relacionados con el medio ambiente.

Las actividades dieron inicio con una multitudinaria marcha de protesta contra el capitalismo, ante la situación ambiental cerca de 30 mil personas, ecologistas, sindicatos, partidos de izquierdas, movimientos de mujeres y homosexuales, pacifistas, hippies y activistas de las más diversas corrientes se unieron en una multitudinaria marcha que inauguró el Foro, el mayor evento de los movimientos sociales del planeta.

"No parimos hijos para la guerra y no queremos ni una guerra que nos mate ni una paz que nos oprima", declaró a la agencia Efe Nelsi Ariza, del movimiento Ruta Pacífica de Mujeres, de Cartagena de Indias. Vestidas de negro, cargaban ataúdes reales que por momentos dejaban en el piso y abrían para mostrar su contenido, que eran cientos de plantas y flores con las que simbolizaban el "entierro de la naturaleza", que atribuyeron al "capitalismo salvaje".

Así también, protestaron contra la construcción de la central hidroeléctrica de Belo Monte, una presa que el Gobierno brasileño levanta en el río Xingú, en la región amazónica, que según los ambientalista, generar daños al ecosistema y a las poblaciones de indios $y$ colonos que habitan en las riberas del Xingú.

Se reflexionó sobre la necesidad de reinventar la convivencia humana. Nos encontramos en un mundo donde se mercantiliza la vida y se destruyen los ecosistemas. Definitivamente. otro mundo debe ser posible, y lo será a partir de la convergencia de todas esas movilizaciones, de los que luchan por la preservación ambiental, del diálogo entre las fuerzas sociales y políticas convencidas de que dentro del capitalismo no hay salvación para el futuro de la humanidad.

Justamente en junio de 2012 se realizará la Conferencia Mundial auspiciada de nuevo por la Organización de las Naciones Unidas, ONU, que conmemora los 20 Años de la realización de la Cumbre de la Tierra Eco 92 que se realizó en la ciudad de Río de Janeiro. Este Foro Social Mundial busca generar un espacio de encuentro entre diversos movimientos sociales alrededor de los temas de la Cumbre Río+20, alrededor de la cual diversas organizaciones y movimientos sociales, han convocado para esas mismas fechas una Cumbre de los Pueblos que busca la movilización mundial para presentar una agenda de transición frente al fracaso de las cumbres oficiales. 
El FSM de Porto Alegre 2012 buscó ser nuevamente el punto de encuentro de personas capaces de reflexionar una salida a la crisis y las bases de la construcción de un nuevo modelo. Los ejes temáticos estuvieron envueltos en cuatro ejes principales: 1) Fundamentos éticos y filosóficos, 2) Derechos humanos, pueblos, territorios y defensa de la Madre Tierra; 3). Producción, distribución y consumo: acceso a la riqueza, bienes comunes y economía de transición, 4) Sujetos políticos, arquitectura de poder y democracia.

Algunos han tildado este último encuentro como un fracaso, por diversos motivos, entre los cuales podemos citar: que es un espacio vital para la reflexión, pero que deja de lado la producción de alternativas y de movilizaciones en contra del injusto orden internacional prevaleciente. Porque los activistas de Porto Alegre no lograron ponerse de acuerdo en una propuesta para el encuentro de la conferencia de la ONU Rio+20, que se celebrará en Rio en junio próximo. Porque se enfrentaron ambientalistas con intelectuales de izquierdas y no hubo consenso. ¿Será realmente un fracaso?
En este sentido, Francisco "Chico" Whitaker $^{14}$ reflexiona sobre la necesidad de cambiar de estrategia e lograr inventar una manera nueva de empezar a hablar al 99\% de insatisfechos del mundo.

¿Cómo concluye el FSM? Desde propuestas de educación popular, pasando por iniciativas de economía cooperativa, a favor de la soberanía alimentaria, alternativas feministas, experiencias contra la privatización de los servicios públicos, de denuncia de la economía verde. Finalizando entre otras con un Ilamado de movilización mundial en junio próximo, para protestar contra la Conferencia de Naciones Unidas Río+20, sobre Desarrollo Sustentable en Río de Janeiro cuyo objetivo es enviar un llamado de alerta a los líderes mundiales sobre el cese al proceso de destrucción del planeta que encabezan las potencias mundiales. Así como, concientizar a toda la sociedad civil del planeta sobre la ausencia de un compromiso real contra el cambio climático que cada vez deteriora en mayor medida el medio ambiente producto en una gran parte de la acción depredadora del ser humano.

\section{Reflexiones finales}

Pareciera, pues, que estamos frente a dos mundos, ¿distintos y distantes?: uno en "Davos" y uno en "Porto Alegre", lo cierto es que las estadísticas muestran lo que está a la vista, por un lado tenemos opu- lencia, pero por otro, mucha necesidad. Primeros y terceros mundos interactuando uno en otro.

El ser humano de Davos, personificado como "el Hombre de 
Davos", expresión que pretende describir al típico o ideal visitante del FEM, frase perpetuada a partir de 2004 por el politólogo norteamericano Samuel Huntington. Se le describe como un hombre, por lo general de raza blanca, bien formado, de mediana edad, de preferencia de origen estadounidense, que no está impregnado de una lealtad nacionalista ni patriotera, ya que considera las fronteras nacionales como un obstáculo y a los gobiernos como superfluos o que están demás.

Félix E. Müller, periodista del Neue Zürcher Zeitung (NZZ), de Suiza, sostiene que la personificación auténtica del "hombre de Davos" ha sido el banquero inversionista que al margen de la política, recorre el mundo infatigablemente en búsqueda de grandes negocios con enormes ganancias. En el pódium de Davos figuran prominentes que dan sus consejos al mundo de como regular y manejar la economía, cómo dirigir empresas y cómo deben estar los Estados organizados.

Reuniones mundiales de empresarios, figuras de la política y comerciales buscan mejorar el estado del mundo, repensarlo, rediseñarlo y reconstruirlo. Sin embargo, la frase de Kenneth Roth, director ejecutivo de la organización Human Rights Watch: "Todo se trató de economía, y todos estuvieron perdidos", nos lleva a la reflexión a pensar: Si tratamos de hablar de economía únicamente enfocándonos en la élite económica y en las crisis financieras, estamos totalmente sesgados con la realidad. La economía es mucho más integrada, que meramente números, como dijo Javier Ibisate: "La economía es una ciencia social que en síntesis estudia el comportamiento de la humanidad en la búsqueda de la satisfacción de sus necesidades humanas (tiene orígenes diferentes) con el uso más eficiente de los recursos limitados de producción".

Estamos seriamente preocupados por la crisis económica, y hemos llegado al punto de creer que lo más importante es el dinero y lo económico en este modo de producción. Como consecuencia esto lleva a minimizar el resto de crisis que están relacionadas con el uso de los recursos de manera desigual y que en definitiva nos hace tener una visión del mundo parcial.

No podemos ofrecer mejorar el estado del mundo, si no tomamos en cuenta las crisis del medio ambiente, desempleo, crecimiento del sector informal, pobreza, hambre, los paraísos fiscales, la violencia entre países y al interior de los países, la exclusión por género, raza y credo, entre otras muchas dificultades que afecta a nuestra sociedad mundial.

Se debe de pensar en un nuevo planteamiento, un nuevo sistema de valores mucho más amplio, 
repensado, rediseñado y reconstruido, que priorice a un mismo nivel de importancia lo económico, lo social, la cultura, lo político y ambiental. Un sistema que retome los tres principios plasmados por Ignacio Ramonet en su artículo "Salvar la tierra". Primero, la precaución que nos ayude a prevenir más que reparar. Segundo, la solidaridad entre las generaciones presentes $y$ futuras; y tercero, la participación: de todos los actores sociales en la toma de decisiones.

Por su parte, "el ser humano de Porto Alegre", es un ser con rostros diferentes, colores y culturas, en busca de un mundo distintos al que existe, posible desde las mayorías populares, que se reúne en países del tercer mundo y que busca evaluar más que la crisis económica como punto central, obligados a reinventar la convivencia humana.

Si realmente se considera que otro mundo es posible, debemos trabajar duro en construirlo (con toda la serie de implicaciones que esto genera), debe ser a través de la convergencia entre lo económico y lo social. Lo que está claro, es que todas las partes deben entablar un diálogo; pero habrá que hacerlo en el contexto de una comprensión de la globalización y del capitalismo, donde debe quedar claro que lo actual debe cambiar, que no es factible en el largo plazo por que produce cada vez más perdedores y menos ganadores
Debe haber un encuentro entre sujetos económicos, políticos y sociales que desarrollen la capacidad de encontrar salidas reales, factibles y de largo plazo para todos y todas. En donde nos enteremos que todos necesitamos de todos y que la práctica del individualismo maximizador permite cavar nuestras tumbas, unas más rápido que otras.

Sin reflexión seria, sin solidaridad, sin cuido del medio ambiente y comercio internacional justo; sin el esfuerzo de todos nosotros, otro mundo es imposible. Todas las personas tenemos el derecho a mejores condiciones de vida en el mundo, pero también todos y todas estamos en el deber de construirlo.

¿Sera que, cómo decía un artículo que leía en el Foro de Davos 2012, que el capitalismo es como un carro viejo arruinado y que solo hace falta comparar unos nuevo? ¿no será que el carro ya no es factible y que necesitamos cambiar a un nuevo medio de transporte que no sea tan costoso como ha sido el capitalismo?

Si el Foro Social Mundial de Porto Alegre se ha considerado agonizante y si el Foro Económico Mundial de Davos, tampoco brilló con excesivos motivos de esperanza, nos indica, que estamos ante un sistema y mundo que muere, y otro que empuja para nacer, es pues necesario sentarnos a reinventar el mundo. 
Recuerdo hace unos meses que leí un artículo escrito por Ignacio Ramonet en Le monde Diplomatique (2012), el cual se titulaba "El año de todos los peligros", en el que comenzaba haciendo una pregunta: ¿será que el 2012 es el año del fin del mundo? Y con honestidad pensé, como respuesta a esta pregunta, ¡esperaría sinceramente que sí!, y que nosotros como académicos y como sociedad fuéramos parte de la destrucción; que fuera el fin del mundo tal como lo conocernos ahora. Y que pudiéramos construir un mundo diferente.

Solo si reconocemos que hemos fracasado, podemos tener la capacidad para iniciar otro camino, una tercera vía. Factible para todos y todas, centrada en al menos dos elementos claves: Primero (una antigua reflexión de las lecciones del padre Ibisate) hacia una reflexión al estilo Perestroika con tres conceptos claves para dicha reflexión, aplica a nivel de país y a nivel mundial, ambos necesarios.

Una Perestroika implica reformas, cambios menores, pero también requiere cambios estructurales. Tal como ilustra Mijaíl Gorbachov, tiene que ver con ser capaces de superar el proceso de estancamiento, de fomentar una verdadera democracia, cambiar los métodos para lograr generar nuevas iniciativas, desarrollar de forma prioritaria la esfera social y los requerimientos particulares de cada región. Esto no vendrá como una especie de revelación, sino como el resultado de un proceso de reflexión seria, formal e inclusiva.

En este sentido son necesarias el "Glasnot que implica decir la verdad" como objetivo y como condición necesaria para las mejoras del mundo, que tiene que ver a su vez con dos cosas, primero con eliminar la corrupción a todo nivel, que la población pueda expresar con la plena libertad y sin temores de su propia verdad, parecer y plantear sus propuestas serias, en donde se puedan construir realmente la senda de la expresión democrática. En segundo lugar, tiene que ver con cómo decir la verdad desde arriba, en donde se elimine la Nomenklatura, el todo saber, el todo poder y el todo imponer.

Y por otra parte es necesario el Novove Mislesnhive, que implica nuevas ideas, para nuestros países y para el mundo, esto esta encadenado a poner fin a toda especie de dogmatismo, desde arriba y poner fin al verticalismo, buscar la construcción de un país y un mundo más horizontal, en donde las autoridades estén cerca de la población y donde se recuperen las confianza y pueda haber esperanza en un nuevo sistema.

Segundo elemento clave: la búsqueda de un nuevo sistema que logre conciliar lo económico, lo social, lo político y lo ambiental, 
cómo pilares fundamentales. Un nuevo modelo que no esté basado en la explotación indiscriminada de la naturaleza y del ser humano, que definitivamente tiene que ver con el esquema de consumo y producción. ¿Cómo? ¿Con cuales herramientas? Esta es la tarea de todas y todos nosotros

Estos dos elementos claves deberán de trabajarse en conjunto, sin duda se convertirá en un proceso mucho más lento y más complicado, pero definitivamente necesario para mejorar el estado del mundo, del presente, del mediano plazo y de las futuras generaciones.

Ese es un poco el objetivo y el espíritu que nos llevo a reflexionar este año en honor al padre Ibsisate, es necesario que podamos analizar el mundo como es hoy, a manera de fotografía desde lo grande números económicos, deliberar acerca de los problemas globales, el endeudamiento creciente de los Estados, la ascendente regulación y desregulación de los mercados, las grandes desigualdades macroeconómicas, el peligro de la sobre-regulación, el recalentamiento climático, las secuelas de la corrup- ción, el impacto de la migración, el estancamiento de los países pobres, el ensanchamiento de la brecha entre ricos y pobres, entre otros; pero por favor, sin olvidar que detrás de esos números estamos hablando de personas, de hombres, mujeres, niños y niñas, Africanos, Asiáticos, Latinoamericanos, de salvadoreños y salvadoreñas. Somos Ilamados a comprometernos desde donde estamos a cambiar el mundo empezando en casa. Que en definitiva pasemos de la protesta a la propuesta.

Muy probablemente en los días que se llevó a cabo la II Jornada de Reflexión Económica Padre Franciso Javier Ibisate, S.J. "Análisis crítico del entorno económico mundial: ciclos y fase histórica del capitalismo" no cambiamos el mundo, pero honramos la memoria del Padre reflexionando en donde estamos y hacia adonde queremos ir, quizá sea hacia una utopía, pero como dice Eduardo Galeano "La utopía está en el horizonte. Camino dos pasos, ella se aleja dos pasos y el horizonte se corre diez pasos más allá. ¿Entonces para qué sirve la utopía? Para eso, sirve para caminar." 


\section{Bibliografía}

- International Business Times. México 28 de Enero 2010.

- http://www.ibtimes.com.mx/articles/20100128/cuanto-cuesta-irdavosforo-economico-mundial-porque-ir.htm

- El Economista. Orígenes del Foro Económico Mundial. 26 de Enero 2009

- http://eleconomista.com.mx/notas-online/davos/2009/01/26/origenesforo-economico-mundial

- BBC Mundo

- http://www.bbc.co.uk/mundo/

- World Economic Forum

- http://www.weforum.org/en/events/AnnualMeeting2010/Sun31/index.htm

- Libertad y Desarrollo. centro de estudios e investigación privado

- http://www.lyd.com/lyd/index.aspx?channel=4358

- La prensa Grafica. El Salvador

- http://www.laprensagrafica.com/economia/internacional/90005-finalizaforo-celebrado-en-suiza.html

- Artículo publicado por la revista especializada Foreign Affairs. El 31 de enero 2011. Puede consultarlo en su versión en Ingles http://relooney. info/0_New_9585.pdf

- Davos 2011: "La economía mundial rumbo a la recuperación". BBC Mundo

- http://www.bbc.co.uk/mundo/noticias/2011/01/110126_ultnot_davos_ economia_mundial_amab.shtml

- World Economic Forum. http://www.weforum.org/events.

- Usted puede encontrar la lista completa de la agenda de FEM y los temas principales tratados durante las reuniones en su versión en Ingles el WEF http://www.weforum.org/events/world-economic-forum-annualmeeting-2011

- BBC Mundo. Davos quiere más mujeres. 13 de Enero 2011.

- http://www.bbc.co.uk/mundo/noticias/2011/01/110113_davos_mujeres_am.shtml 
- La Nación. Cl. ¿Está el mundo maduro para la Mujer de Davos? 30 de Enero 2011

- $\quad$ http://www.lanacion.cl/-esta-el-mundo-maduro-para-la-mujer-de-davos-/ noticias/2011-01-28/172455.html

- New York Times

- http://www.nytimes.com/

- http://dealbook.nytimes.com/2011/01/24/a-hefty-price-for-entry-to-davos/

- Claves: un foro económico mundial BBC Mundo 2005

- http://news.bbc.co.uk/hi/spanish/international/ newsid_3416000/3416351.stm

- $\quad$ Davos quiere más mujeres BBC Mundo 2011

- http://www.bbc.co.uk/mundo/noticias/2011/01/110113_davos_mujeres_am.shtml

- $\quad$ The World Economic Forum Leadership Team

- http://www.weforum.org/content/leadership-team

- $\quad$ Lo que dejó Davos 2011. BBC Mundo 2011

- $\quad$ http://www.bbc.co.uk/mundo/noticias/2011/01/110131_davos_2011_ conclusiones_pl.shtml?print=1

- $\quad$ Ramonet Ignacio (2012) El año de todos los peligros

- http://www.monde-diplomatique.es/?url=editor i a l/ $0000856412872168186811102294251000 /$ editorial/?articulo=f62c3145-18d7-4a1c-a93f-3f42321999b0

- World Economic Brainstorming: Business, Governance and Leadership - New Pressures and Realities - Annual Meeting 2012

- $\quad$ http://www.weforum.org/videos/world-economic-brainstorming-businessgovernance-and-leadership-new-pressures-and-realities-a

- Davos 2012: los magnates se preguntan si el capitalismo tiene futuro

- $\quad$ http://www.bbc.co.uk/mundo/noticias/2012/01/120124_para_que_sirve_otra_reunion_davos_jr.shtml

- Davos 2011: "La economía mundial rumbo a la recuperación"

- http://www.bbc.co.uk/mundo/noticias/2011/01/110126_ultnot_davos_ economia_mundial_amab.shtml 
- Policía suiza presenta cargos contra manifestantes anti: Davos Reporte de Silke Koltrowitz. Editado en español por Marion Giraldo.

- $\quad$ http://Ita.reuters.com/article/worldNews/idLTASIE80M00520120123

- Edelman

- http://www.edelman.com/citizenship/

- $\quad$ Video de Davos 2012

- $\quad$ http://www.weforum.org/videos/davos-2012-great-transformation-shaping-new-models

- Conference: Eurozone Must Show "Colour of Money" before World Helps with Financial Rescue. WEF. Davos-Klosters, Switzerland, 28 January 2012.

- http://www.weforum.org/news/eurozone-must-show$\%$ E2\%80\%9Ccolour-money\%E2\%80\%9D-world-helps-financial-rescue

- CNBC Debate: Who's in Charge? Ibrahim S. Dabdoub Angel Gurría Nandan M. Nilekani John W. Snow George Soros Joseph E. Stiglitz Moderated by Maria Bartiromo WEF. Wednesday 23 January

- $\quad$ http://www.weforum.org/sessions/summary/cnbc-debate-whos-charge

- $\quad$ FEM. Global Risks 2012: The Seeds of Dystopia. Wednesday 25 January

- $\quad$ http://www.weforum.org/sessions/summary/global-risks-2012-seedsdystopia

- FEM. Davos Debate on Globalization Thursday 26 January

- $\quad$ http://www.weforum.org/sessions/summary/davos-debate-globalization

- Davos 2012: Business needs to build trust

- $\quad$ http://www.weforum.org/content/davos-2012-business-needs-build-trust

- FEM: 25 de Enero 2012. To Serve Society Better, Capitalism Needs a Redesign

- $\quad$ http://www.weforum.org/news/serve-society-better-capitalism-needsredesign

- $\quad$ TIME Davos Debate on Capitalism - Annual Meeting 2012

- http://www.weforum.org/videos/time-davos-debate-capitalism-annualmeeting-2012

- Davos 2012 Co-Chair - Paul Polman

- $\quad$ http://www.weforum.org/videos/davos-2012-co-chair-paul-polman

- $\quad$ http://www.weforum.org/sessions/summary/global-agenda-2012 
- $\quad$ Ensuring Food Security - Annual Meeting 2012

- http://www.weforum.org/videos/ensuring-food-security-annual-meeting-2012

- Paginal official del Foro Social mundial

- Biografía de Bernard Cassen

- $\quad$ http://www.fundacionmondiplo.es/biocassen.htm

- El consenso de Porto Alegre. Ignacio Ramonet, director de Le Monde Diplomatique y uno de los fundadores de ATTAC. Escrito en "El País" (2 de Febrero de 2001)

- http://www.nodo50.org/derechosparatodos/Areas/AreaFSocial4.htm

- Foro Social Mundial de Porto Alegre 2001.LLAMADO A LA MOVILIZACION

- http://latinoamericana.org/2002/textos/castellano/ForoSocialMundial.htm

- El Comité Organizador del Foro Social Mundial de Porto Alegre divulga resoluciones

- $\quad$ http://www.nodo50.org/derechosparatodos/Areas/AreaFSocial6.htm

- Seminário internacional 10 Anos Depois: Desafios e propostas para um outro mundo possível

- $\quad$ http://seminario10anosdepois.wordpress.com/debates/seminario-10-anosdepois-25-a-29-de-janeiro/

- Foro Social Mundial 2010 tendrá programación descentralizada

- http://www.adital.com.br/site/noticia.asp?lang=ES\&cod=43525

- Fórum Social mundial 10 años.

- http://fsm10.procempa.com.br/wordpress/

- Sergio Ferrari (2011) desde Dakar, Senegal. RADIOGRAFIA DE UN PROCESO PLANETARIO EN CRECIMIENTO

- $\quad$ http://rebelion.org/noticia.php?id=121788

- Comenzó Foro Social Mundial en Dakar con multitudinaria marcha (2011)

- $\quad$ http://www.quiendebeaquien.org/spip.php?article2065

- $\quad$ Foro Social Mundial 2011 DAKAR

- http://fsm2011.org/es/noticias/fsm-2011-dia-8-de-febrero-martes

- África convoca al planeta a construir alternativas 2011

- $\quad$ http://rebelion.org/noticia.php?id=121788 
- Carta de solidaridad con la lucha de las mujeres del mundo

- http://fsm2011.org/es/noticias/carta-de-solidaridad-con-la-lucha-de-lasmujeres-del

- FSM 2011 -Dakar- Una semana para cambiar el mundo

- http://unasemanaparacambiarelmundo.blogspot.com/

- Declaración de la Asamblea de los Movimientos Sociales del FSM 2011 (febrero de 2011)

- http://www.quiendebeaquien.org/spip.php?article2071

- Cobertura. FSM 2011*

- http://fsm2011.org/es/coberturas

- Foro Social Mundial 2012

- http://www.cubadebate.cu/opinion/2012/01/14/foro-social-mundial-2012/

- Ferrari Sergio (2012) Francisco Chico Whitaker: "El Foro Social Mundial sigue vivo" http://www.argenpress.info/2012/02/francisco-chicowhitaker-el-foro-social.html

- Santana Rodríguez, Pedro (2012) "Los motivos de una convocatoria: Foro Social Mundial en Porto Alegre 2012"

- http://alainet.org/active/52275\&lang=es

- Multitudinaria marcha contra el capitalismo abre el Foro Social Mundial

- http://informe21.com/politica/12/01/24/multitudinaria-marcha-contra-elcapitalismo-abre-el-foro-social-mundial

- Masiva marcha en el comienzo del Foro Social Mundial

- http://www.pagina12.com.ar/diario/ultimas/20-186132-2012-01-24.html

- Carlos Casuso, Investigación e Innovación Método Hélmer (2012). Foro Social Mundial 2012, del 24 al 29 de enero

- http://blog.metodohelmer.com/2012/01/23/foro-social-mundial-2012-del24-al-29-de-enero/

- Foro Social Mundial culmina con Ilamado a protesta global el 5 de junio

- 29 de Enero de 2012.

- http://mesaredonda.cubadebate.cu/noticias/2012/01/29/foro-socialmundial-culmina-con-Ilamado-a-protesta-global-el-5-de-junio/ 
7 FEM To Serve Society Better, Capitalism Needs a Redesign Wednesday 25 January 2012.

8 FEM Davos 2012 Co-Chair - Paul Polman.

9 Ensuring Food Security - Annual Meeting 2012.

10 Cassen era entonces el director de Le Monde Diplomatique, fundador y primer presidente de ATTAC Francia (1998-2002)

11 Foro Social Mundial de Porto Alegre 2001.Llamado a la movilización

12 Ferrari Sergio (2011) Radiografía de un proceso planetario en crecimiento.

13 Carlos Casuso, Investigación / Innovación Método Hélmer (2012). Foro Social Mundial 2012, del 24 al 29 de enero.

14 Arquitecto brasileño, uno de los cofundadores del FSM, quien recibió en 2006 el Premio Nobel alternativo por su compromiso altermundialista activo. 